\title{
Oxime Type Photoacid Generators Having Adamantyl and Superstrong Acid Precursor Group
}

\author{
Masumi Yamamoto and Shigeru Takahara* \\ Department of Materials Science, Graduate School of Science and Engineering, Chiba University, \\ 1-33 Yayoicho, Inage-ku, Chiba 263-8522, Japan \\ *takahara@faculty.chiba-u.jp
}

\begin{abstract}
Oxime derivatives, having a N-O bond in the molecule structure, are excellent photo reactive compounds. Oxime type photoacid generaters with adamantyl and perfluoroalkylsulfonyloxy groups and without aromatic groups were prepared, and their absorption spectra and thermal properties were measured. The compounds designed with photoacid generator in deep ultraviolet (DUV) region have good transparency in the region. The thermal stability of these PAGs depended on the chain length of acid precursor groups. The quantum yields of acid generation were substantially high. These aliphatic oxime sulfonates can be expected to be PAGs in DUV. Keywords: Photo-acid generator, Oxime ester, Adamantane, Chemically amplified photoresist, Superstrong acid
\end{abstract}

\section{Introduction}

Oxime derivatives are useful photo-reactive compounds for photo-acid generators (PAGs) [1] and other photo-initiators [2,3], which are initiated by $\mathrm{N}-\mathrm{O}$ bond cleavage in high quantum yield. We have focused the reactions and sensitization of oxime type PAGs [4-8]. Oxime type PAGs have been attempted to be applied to i-line, $\mathrm{KrF}(248$ $\mathrm{nm})$ and $\operatorname{ArF}(193 \mathrm{~nm})[9,10]$ and 157-nm [3] lithography. In the reference [3], an oxime type PAG, (Z,E)-2-(4-methoxyphenyl)-2-[(4-methylphenylsulfonyl)oxyimino]acetonitrile (PAIOTos) was evaluated not to perform high resolution because it generates 4-methylbenzenesulfonic acid, which is not a superstrong acid like perfluoroalkylsulfonic acid. PAGs to generate superstrong acid are significant players in photopolymer science. Up to the present date, the PAGs have been developed from various aspects. For example, the solubility was designed by computational method [11], or they act as polymer-bound PAGs [12-14]. The PAGs to generate superstrong acid were also used for UV curing [15] or in chemical amplification [16]. A new acid generation mechanism was also proposed [17]. On the other hand, for the light source of deep ultraviolet (DUV) region, the PAG molecule does not need to have an aromatic group to be excited. Base polymers designed for the ArF resist have aliphatic structures with alicyclic groups. A PAG, generating a superstrong acid containing adamantyl group, was reported as a polymer-blended PAG with an aliphatic base polymer [13]. However, it was a triphenylsulfonium salt, and there were few reports of aliphatic PAGs.

We focused and proposed some oxime sulfonates having adamantyl group [18] to provide high sensitivity and to control transparency. In the report, $\quad(Z, E)-2-(1$-adamantyl)-2-[((4-methylphenyl)sulfonyl)oxyimino]acetonitrile (ACIOT) having acid precursor with an aromatic group showed a good thermal stability and gave a good sensitivity with a base polymer. However, (Z,E)-2-(1-adamantyl)-2-[((trifluoromethyl)sulfonyl )oxyimino]acetonitrile (ACIOF) containing no aromatic group showed very poor quantum yield of acid generation $\left(\phi_{a c i d}\right)$ and low thermal stability, but not bad sensitivity as photopolymer material. The behaviors were confusing for us. The structure like ACIOF containing alicyclic groups without aromatic group was thought to have some 
advantages, such as affinity with aliphatic base polymers. Then, we have tried to prepare the series of aliphatic PAGs including ACIOF again.

Here, we prepared purified ACIOF by modified method, and corrected the data of $\phi_{a c i d}$ and thermal properties. To confirm the potential, we also prepared some aliphatic PAGs to generate perfluoroalkylsulfonic acids with a different alkyl chain length from that of ACIOF.

\section{Materials and methods}

\subsection{Materials}

Oxime type PAGs containing adamantyl group and perfluoroalkylsulfonyloxy groups, $(Z, E)-2-(1$-adamantyl)-2-[((nonafluorobutyl)sulfony 1)oxyimino]acetonitrile

(ACIOB), (Z,E)-2-(1-adamantyl)-2-[((heptadecaoctyl)sulfonyl )oxyimino]acetonitrile (ACIOP), and ACIOF, were prepared. Figure 1 shows their molecular structures.

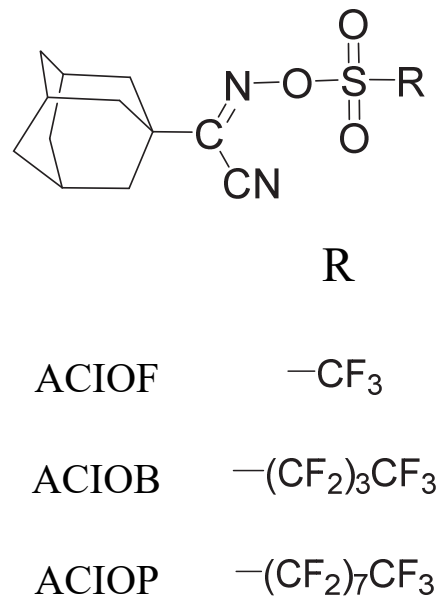

Fig. 1. Chemical structures of oxime type PAGs having adamanyl and perfluoroalkylsulfonyloxy groups.

\subsubsection{Synthesis of ACIOF}

In the previous report [18], ACIOF was prepared from 1-adamantyl-2-hydroxyiminoacetonitrile and trifluoromethanesulfonic acid. We modified the method to use lithium trifluoromethanesulfonate instead of trifluoromethanesulfonic acid. After the preparation of 1-adamantylamphiglyoxime [19] and 1-adamantyl-2-hydroxyiminoacetonitrile [20] by the method as described in [18], the mixtures of 1-adamantyl-2-hydroxyiminoacetonitrile $(0.16 \mathrm{~g}$, $\left.7.84 \times 10^{-4} \mathrm{~mol}\right), 3 \mathrm{~mL}$ trimethylamine and $50 \mathrm{~mL}$ tetrahydrofuran (THF) were stirred. Then, lithium trifluoromethanesulfonate $\left(0.12 \mathrm{~g}, 7.84 \times 10^{-4} \mathrm{~mol}\right)$ (Tokyo Chemical Industry Co.) was added and stirred overnight at $5{ }^{\circ} \mathrm{C}$. After a large amount of distilled water was poured into the solution, the solvent was removed in rotary evaporator. Then, the residual was dissolved in chloroform again, and the solution was washed with distilled water in a separation funnel. After that, the solvent was removed to give yellow-white precipitate and it was dried in vacuum overnight. The yield was $49 \%$ $\left(0.15\right.$ g). ${ }^{1} \mathrm{H}-\mathrm{NMR} \quad\left(\mathrm{CDCl}_{3}, \quad 400 \mathrm{MHz}\right): \quad \delta$ $1.682-2.074(\mathrm{~m}, 15 \mathrm{H})$.

\subsubsection{Synthesis of ACIOB}

ACIOB was prepared from 1-adamantyl-2-hydroxyiminoacetonitrile and lithium nonafluoro-1-butanesulfonate $(0.24 \mathrm{~g}, 7.84$ $\times 10^{-4} \mathrm{~mol}$ ) (Tokyo Chemical Industry Co.) under the same conditions as the preparation of ACIOF. The yield was $39 \%(0.13 \mathrm{~g}) .{ }^{1} \mathrm{H}-\mathrm{NMR}\left(\mathrm{CDCl}_{3}, 400\right.$ MHz): $\delta 1.682-2.079(\mathrm{~m}, 15 \mathrm{H})$.

\subsubsection{Synthesis of ACIOP}

ACIOP was prepared from

1-adamantyl-2-hydroxyiminoacetonitrile and lithium heptadecafluoro-1-octanesulfonate $(0.39 \mathrm{~g}$, $7.84 \times 10^{-4} \mathrm{~mol}$ ) (Tokyo Chemical Industry Co.) under the same conditions as the preparation of ACIOF. The yield was $48 \%(0.15 \mathrm{~g}) .{ }^{1} \mathrm{H}-\mathrm{NMR}$ $\left(\mathrm{CDCl}_{3}, 400 \mathrm{MHz}\right): \delta 1.685-2.082(\mathrm{~m}, 15 \mathrm{H})$.

\subsection{Thermal analysis}

About $10 \mathrm{mg}$ of the sample was weighed into an aluminum pan, and the TG-DTA measurement (DTG-60, Shimazu Co.) was carried out at heating rate of $10{ }^{\circ} \mathrm{C} \mathrm{min}{ }^{-1}$ under nitrogen.

\subsection{Quantum yield of acid generation ( $\left.\phi_{\text {acid }}\right)$}

Measurement of photoacid generation quantum yield was carried out as described in the previous paper [21]. The PAG solutions were exposed to the light of $222 \mathrm{~nm}$ in wavelength from an excimer lamp (USHIO UER20-222) here.

\section{Result and discussion}

We successfully prepared three alicyclic oxime sulfonates for PAG. ACIOF was designed to generate trifluoromethanesulfonic acid, and ACIOB generates nonafluorobutanesulfonic acid, and ACIOP generates heptadecafluorooctanesulfonic acid, known as superstrong acids.

\subsection{Absorption spectra of PAGs in solution}

The UV-Vis absorption spectra of the PAGs in acetonitrile are shown in Fig. 2. ACIOF, ACIOB, and ACIOP showed very small absorption in the wavelength region over $250 \mathrm{~nm}$ in comparison with 
PAIOTos, which has aromatic groups in the molecule. The absorption peaks of three alicyclic oxime sulfonates between 200-250 $\mathrm{nm}$ were slightly shifted to short wavelength according to the chain length of perfluoroalkyl groups. Their molecular extinction coefficients were more or less half that of PAIOTos. These alicyclic PAGs contain adamantyl group, and therefore, seem to be suitable for DUV lithography due to their high transparency in the region.

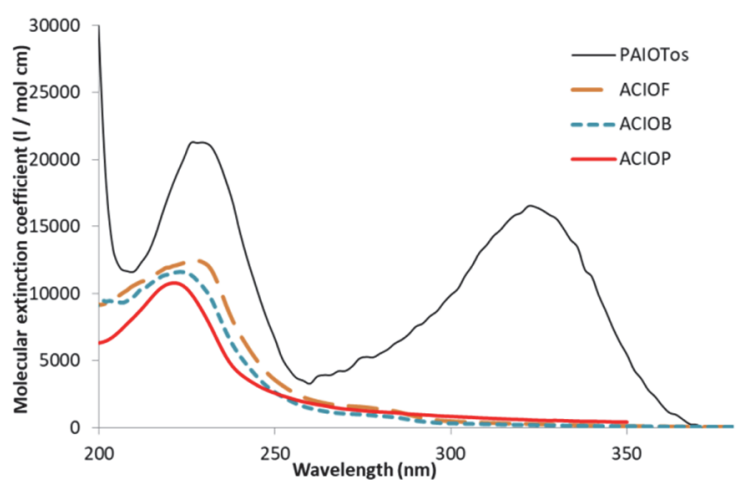

Fig. 2. Absorption spectra of ACIOF, ACIOB, ACIOP, and PAIOTos [18] in acetonitrile.

3.2. Quantum yield of acid generation $\left(\phi_{a c i d}\right)$ and thermal properties of PAGs

By the irradiation of $222 \mathrm{~nm}$ light, the $\phi_{\text {acid }}$ of PAGs in acetonitrile solution was measured by a color change reagent, and these are summarized in Table 1. The $\phi_{\text {acid }}$ values of ACIOF, ACIOB and ACIOP were $0.40,0.39$ and 0.46 respectively. They were around 0.40 , and we consider these are reasonable and valid values by the experiments of a series of aliphatic oxime sulfonates. The $\phi_{a c i d}$ values were roughly three times that of PAIOTos [18]. Thus, these oxime sulfonates without aromatic group can be expected to give a high sensitivity in the DUV resists.

The thermal analysis of these aliphatic oxime derivatives was measured under nitrogen, and the respective melting $\left(T_{m}\right)$ and decomposition $\left(T_{d}\right)$ points were also summarized in Table 1. As shown in the table, $T_{m}$ and $T_{d}$ of ACIOF and ACIOB were almost the same. These alicyclic oxime sulfonates exhibit a high $T_{d}$ over $180^{\circ} \mathrm{C}$. The $T_{m}$ and $T_{d}$ values of ACIOP, having long perfluoroalkyl group, were higher than those of ACIOF, ACIOB and even PAIOTos. Those PAGs are considered to have sufficient thermal stability as resist material. Among them, ACIOP has better thermal stability, which has lager molecular weight with a greater number of $-\mathrm{CF}_{2}$-group.

The $T_{m}, T_{d}$ and $\phi_{\text {acid }}$ values of ACIOF reported in a previous report [18] were corrected here. The design of the PAGs, oxime sulfonates having adamantyl group and perfluoroalkylsulfonyloxy groups, achieved good thermal stability and efficiency of superstrong acid generation.

Table 1. Quantum yield of acid generation $\left(\phi_{a c i d}\right)$ in acetonitrile by irradiation of $222 \mathrm{~nm}$ light and thermal properties $\left(T_{m}\right.$ : melting point, $T_{d}$ : decomposition point) under $\mathrm{N}_{2}$ atmosphere.

\begin{tabular}{llll}
\hline PAG & $\phi_{\text {acid }}$ & $T_{m}\left({ }^{\circ} \mathrm{C}\right)$ & $T_{d}\left({ }^{\circ} \mathrm{C}\right)$ \\
\hline ACIOF & 0.40 & 160 & 186 \\
ACIOB & 0.39 & 162 & 189 \\
ACIOP & 0.46 & 255 & 295 \\
PAIOTos [18] & 0.14 & 140 & 273
\end{tabular}

\section{Conclusion}

We succeeded in syntheses of novel oxime type PAGs having adamantyl group and photo-generating perfluoroalkylsulfonic acids. A series of aliphatic oxime esters, ACIOF, ACIOB, and ACIOF have good transparency in the DUV region. In addition, they have sufficient thermal stability as resist material. They can be expected to be highly sensitive PAGs for the resist in $193 \mathrm{~nm}$ lithography. We are evaluating characteristic properties of the resist materials, including these aliphatic oxime type PAGs.

\section{References}

1. T. Hagiwara, S. Irie, T. Itani, Y. Kawaguchi, O. Yokokoji, and S. Kodama, J. Photopolym. Sci. Technol., 16 (2003) 557.

2. A. Reiser, "Photoreactive Polymer", John Wiley and Sons, New York (1989) p112.

3. J. V. Crivello and K. Dietliker, "Photoinitiators for Free Radical, Cationic and Anionic Photopolymerization", 2nd edition, G. Bradley Ed., John Wiley and Sons, New York (1988) p494.

4. S. Takahara, S. Suzuki, T. Tsumita, X. Allonas, J. P. Fouassier, and T. Yamaoka, J. Photopolym. Sci. Technol., 21 (2008) 499.

5. S. Takahara, N. Nishizawa, and T. Tsumita, $J$. Photopolym. Sci. Technol., 22 (2009) 289.

6. S. Suzuki, J. Iwaki, T. Urano, S. Takahara, and T. Yamaoka, Polym. Adv. Technol., 17 (2006) 348.

7. J.-P. Malval, S. Suzuki, F. Morlet-Svary, X. Allonas, J.-P. Fouassier, S. Takahara, and T. Yamaoka, Chem. Phys. Lett., 443 (2007) 323. 
8. J.-P. Malval, S. Suzuki, F. Morlet-Svary, X. Allonas, S. Takahara, and T. Yamaoka, J. Phys. Chem. A, 112 (2008) 3879.

9. T. Asakura, H. Yamato, T. Itani, Y. Nishimae, and M. Ohwa, J. Photopolym. Sci. Technol., 20 (2007) 465.

10. H. Yamato, T. Asakura, Y. Nishimae, A. Matsumoto, J. Tanabe, J. Birbaum, P. Murer, T. Hintermann, and M. Ohwa, J. Photopolym. Sci. Technol., 20 (2007) 637.

11. E. Ryu, M. Kim, Y. Yoon, K. Im, H. Jeong, H. Jeon, H. Lee, and H. Kim, J. Photopolym. Sci. Technol., 29 (2016) 731.

12. C. Lee, C. L. Henderson, M. Wang, K. E. Gonsalves, and W. Yueh, J. Vac. Sci. Technol. B, 25 (2007) 2136.

13. H. Yamamoto, T. Kozawa, and S. Tagawa, Microelectron. Eng., 129 (2014) 65.
14. S. Nandi, M. Yogesh, P. G. Redd, S. K. Sharma, C. P. Pradeep, S. Ghosh, and K. E. Gonsalves, Mater. Chem. Front., 1 (2017) 1895.

15. M. Shirai and H. Okamura, Prog. Org. Coat., 64 (2009) 175.

16. K. Arimitsu, M. Yonokura, and M. Furutani, RSC Adv., 5 (2015) 80311.

17. R. Li, T. Nakashima, and T. Kawai, Chem. Commun., 53 (2017) 4339.

18. S. Wada, Y. Yagi, K. Hase, K. Nakajima, and S. Takahara, J. Photopolym. Sci. Technol., 28 (2015) 55.

19. I. K. Moiseeva, M. I. Kalinina, M. N. Zemtsova, and P. L. Trakhtenberg, Zhurna Organicheskoi Khimii, 22 (1986) 2292.

20. M. I. Kalinina and I. K. Moiseev, Zhurnal Organicheskoi Khimii, 23 (1987) 2571.

21. S. Suzuki, X. Allonas, J. P. Fouassier, T. Urano, S. Takahara, and T. Yamaoka, J. Photochem. Photobiol. A: Chem., 181 (2006) 60. 